\title{
« SOUTIEN ORGANISATIONNEL PERÇU, IMPLICATION ORGANISATIONNELLE ET SATISFACTION AU TRAVAIL : EFFETS SUR L'ABSENTEISME DANS LA FONCTION PUBLIQUE TERRITORIALE ॥
}

\author{
Fatéma SAFY-GODINEAU \\ Maître de Conférences en sciences de gestion \\ Université de Pau et des Pays de l'Adour, \\ Chaire Optima/CREG-MET/IAE Pau-Bayonne \\ fatéma.safy-godineau@univ-pau.fr
}

\author{
Amar FALL \\ Maître de Conférences en sciences de gestion \\ Université de Pau et des Pays de l'Adour, \\ Chaire Optima/CREG-MET/IAE Pau-Bayonne \\ amar.fall@univ-pau.fr
}

\begin{abstract}
David CARASSUS
Professeur des universités en sciences de gestion Université de Pau et des Pays de l'Adour, Chaire Optima/CREG-MET/IAE Pau-Bayonne david.carassus@univ-pau.fr
\end{abstract}

\begin{abstract}
Résumé : Notre étude s'interroge sur les effets du soutien organisationnel perçu, de l'implication organisationnelle et de la satisfaction au travail sur l'absentéisme des agents territoriaux, dans un contexte d'innovation publique. Les résultats de la recherche montrent qu'une faible perception du soutien organisationnel, une forte implication organisationnelle calculée, ainsi qu'une faible implication organisationnelle affective associée à une faible satisfaction au travail, sont les conditions dans lesquelles l'absentéisme des agents territoriaux est le plus probable. Ils soulignent que l'absentéisme des agents territoriaux est à la fois le résultat de leur désaffection envers leur organisation et de l'émergence d'attitudes négatives basées sur des calculs opportunistes. Cette recherche met ainsi en évidence le fait que le soutien organisationnel perçu est un levier potentiel de la lutte contre l'absentéisme et attire l'attention des managers publics sur les effets contre-productifs des pratiques d'incitations financières sur l'absentéisme. Elle montre la nécessité, pour lutter efficacement contre l'absentéisme d'aller vers des pratiques de management éthique favorisant un environnement de travail soutenant dans lequel les agents territoriaux peuvent s'impliquer.
\end{abstract}

Summary : Our study examines, in public innovation context, the effects of perceived organizational support, organizational commitment and job satisfaction on absenteeism among public territorial employees. Research results show that low perception of organizational support, high calculated organizational commitment, as well as low affective organizational commitment associated with low job satisfaction, are the conditions under which absenteeism among public territorial employees is most likely. They emphasize that the absenteeism of public territorial agents is the result both of their disaffection towards their organization and of the emergence of negative attitudes based on opportunistic calculations. This research highlights the fact that perceived organizational support is a potential lever in the fight against absenteeism and draws public managers' attention to the counter-productive effects of financial incentive practices on absenteeism. It shows the need, in order to fight effectively against absenteeism, to move towards ethical management practices favouring a supportive working environment in which public local employees can become committed.

Mots-clés : absentéisme, soutien organisationnel perçu, implication organisationnelle, satisfaction au travail, fonction publique territoriale 


\section{INTRODUCTION}

Les réformes de modernisation du service public, amorcées depuis les années 1980, placent les collectivités territoriales au cœur d'une nouvelle régulation publique. Exigence, efficacité, efficience, performance et adaptabilité constituent dès lors les maîtres-mots d'une nouvelle gestion publique (Emery \& Giauque, 2005), obligeant les collectivités territoriales à faire preuve d'innovation afin de relever simultanément le défi de la maîtrise budgétaire de leur dépense de fonctionnement et de l'amélioration continue de la qualité du service public, dans un contexte de raréfaction de leurs ressources et d'accroissement de leurs compétences (Bartoli, 2006). Or, parmi la diversité des innovations publiques, les innovations de type organisationnel et managérial semblent dominantes dans les organisations publiques (Chomienne \& Pupion, 2009). Flexibilité, mutualisation, fusion, évaluation de la performance publique, rémunération au mérite, management par objectif : autant de processus marquant ainsi l'évolution des modes de fonctionnement, de l'organisation interne et des techniques de gestion des collectivités territoriales (Laoukili, 2009). Bien que nécessaire, ces innovations déçoivent pourtant les espoirs quant aux résultats escomptés. D'une part, elles sont productrices d'antagonismes avec lesquels les managers publics ont mal à composer (Martinet et Payaud, 2006). Cohabitent ainsi ensemble centralisation et transversalité/participation, contrôle et autonomie/responsabilité, commandement et organisation/animation des différents acteurs partie prenante de l'action publique (Bartoli \& Chomienne, 2011), organisation bureaucratique et organisation «bureauganique » (Bouchikhi \& Kimberly, 1992). D'autre pat, elles sont sources d'incertitudes, de résistances, d'insatisfaction des agents territoriaux (Emery \& Giauque, 2012). Dans cette veine, le malaise grandissant des agents territoriaux ${ }^{1}$, de même que la hausse de l'absentéisme dans la fonction publique territoriale ${ }^{2}$, nécessitent de se poser la question du coût humain de l'innovation et, plus spécifiquement, du rôle des cadres publics dans la mise en œuvre et l'accompagnement au changement (Brouwers et al., 1997 ; Damanpour \& Schneider, 2006 ; 2008).

La compréhension de l'absentéisme apparaît alors comme un sujet d'importance pour la Gestion des Ressources Humaines (GRH : Harrison et Martocchio, 1998) dans le cadre des changements territoriaux. D'une part, car la gestion de l'absentéisme relève des missions de la GRH en vue d'assurer le maintien de l'efficacité organisationnelle (Goetzel et al., 2004). D'autre part, car l'absentéisme, considéré comme un comportement contre-productif influençant négativement la performance individuelle des travailleurs (Spector \& Fox, 2002 ; Dalal, 2005), peut être le reflet d'un dysfonctionnement des pratiques de GRH. Dans cette perspective, le lien entre la GRH et l'efficacité organisationnelle est indirecte, par le biais des attitudes et comportements favorables à la performance individuelle (Delery, 1998). Or, il apparaît que ce sont les pratiques et actions de la GRH qui influencent ces attitudes et comportements au travail, générant une amélioration ou une détérioration de la performance individuelle et indirectement de l'efficacité organisationnelle (Edgar \& Geare, 2005).

Au regard de ces développements, notre étude s'intéresse aux effets sur l'absentéisme de trois variables contingentes aux pratiques d'implication et de fidélisation des ressources humaines. Dans ce cadre, nous répondons ainsi à la question suivante : Quels sont les effets du soutien organisationnel perçu, de la satisfaction au travail et de l'implication organisationnelle sur l'absentéisme?

\footnotetext{
${ }^{1}$ Enquêtes « Bien-être au travail dans la fonction publique territoriale » $(2014,2015,2016)$

2 Sofaxis (2016), « Panorama des absences au travail pour raisons de santé dans les collectivités territoriales en $2015 »$; ADRH GCL (2017), « Résultats du benchmark absentéisme 2015 »
} 


\section{1) CADRE CONCEPTUEL, THEORIQUE ET HYPOTHESES DE RECHERCHE}

Objet de recherche fécond, en raison des coûts financiers et des dysfonctionnements qu'il impose aux organisations, l'absentéisme est défini comme un "phénomène polysémique » en raison de la diversité de ses causes, de ses formes, et du sens qui lui est attribué (Johns, 1997). C'est ainsi qu'il est perçu comme un concept ambigu, difficile à mesurer, à comprendre, et traversé par d'innombrables contradictions conceptuelles, théoriques et empiriques (Steel, 2003). Afin de mieux en comprendre les contours, nous nous attèlerons dans cette partie à clarifier le concept d'absentéisme, avant de développer les deux approches théoriques de l'absentéisme communément admises en sciences de gestion: 1'approche individuelle et psychosociale (Bouville, 2009).

\section{1) Clarification conceptuelle de l'absentéisme}

A ce propos, Whitston et Edwards (1990 : 288) notent que "la distinction entre absence et absentéisme est importante et négligée ". L'absence se caractérise comme un évènement ponctuel et normal de la vie de travail, associée aux congés réglementaires (congés payés, congés de formation, congé maternité, congés spéciaux, etc. : Whitston et Edwards, 1990). Au contraire, l'absentéisme se caractérise comme un comportement chronique, répétitif, volontaire, habituel et systématique de manque d'assiduité sur son lieu de travail (Huver, 2013). Plus spécifiquement, il désigne le fait de ne pas être au travail pour des raisons de maladie (Bouville, 2009). Bien que cette distinction soit posée, de nombreuses confusions conceptuelles règnent dans les études sur l'absentéisme, en raison notamment de son opérationnalisation (Blau et Boal, 1987 ; Johns, 2003). En effet, l'absentéisme est mesuré dans les recherches académiques à partir de deux mesures : la fréquence et la durée des absences (Steel, 2003). La fréquence traduit les occurrences d'absence sur une période déterminée, quelque soit le motif de l'absence. La durée traduit, elle, le temps perdu, en jours ou en heures, pendant lequel un salarié s'absente, quelque soit le motif de l'absence (Sagie, 1998). Plus spécifiquement, la fréquence des absences est assimilée à une mesure indirecte de l'absence volontaire, tandis que la durée des absences est perçue comme une mesure indirecte de l'absence involontaire (Chadwick-Jones et al., 1982). Cette distinction du caractère volontaire ou involontaire de l'absentéisme peut se comprendre à travers la typologie établie par Chatterji et Tilley (2002) : l'absentéisme désigne le fait de rester chez soi en étant en bonne santé (absence volontaire), tandis que l'absence désigne le fait de rester chez soi pour un problème de santé (absence involontaire). Si cette typologie permet de mettre l'accent sur les différentes dérives de l'usage des congés maladie, elle contribue toutefois à accentuer la confusion conceptuelle entre absence et absentéisme. En outre, la distinction entre le caractère volontaire et involontaire de l'absentéisme a fait l'objet de nombreuses critiques. Parmi les plus importantes, nous relevons celles qui soulignent que les deux mesures indirectes de l'absentéisme (fréquence vs durée) sont indépendantes du motif des absences. Ce qui induit, au-delà du maintien de la confusion conceptuelle entre absence et absentéisme, une appréciation du caractère volontaire ou involontaire de l'absentéisme reposant davantage sur un jugement de valeur plus que sur une analyse fiable des motifs de l'absentéisme (Nicholson, 1977 ; Brooke \& Price, 1989 ; Alexanderson, 1998). C'est ainsi que plusieurs chercheurs soulignent la nécessité de tenir compte des motifs d'absence, en sus de la combinaison des deux mesures de fréquence et de durée des absences, pour distinguer le caractère volontaire ou involontaire de l'absentéisme ou établir une mesure globale de l'absentéisme (Sagie, 1998 ; Smulders, 1980 ; Steel, 2003).

$\mathrm{Au}$ regard ces critiques, nous ne tiendrons pas compte du caractère volontaire ou involontaire de l'absentéisme dans notre étude. En revanche, nous maintiendrons la distinction entre 
absence et absentéisme. L'absentéisme sera entendu ici comme «la non présence physique d'un individu à un endroit donné et à un moment donné alors qu'on attend de lui qu'il soit là » (Harrison et Price, 2003 : 204). Selon cette définition, qui fait largement consensus dans les travaux académiques, l'absentéisme se caractérise alors par deux éléments : l'absence d'un individu à son poste de travail et le non respect de l'attente sociale qui le concerne. Ce dernier élément permet de distinguer l'absentéisme, entendu comme une absence sur son lieu de travail pour raison de maladie, hors congé maternité ${ }^{3}$, de l'absence autorisée qui est liée aux congés règlementaires. Ainsi, un niveau d'absence élevé ne traduit pas nécessairement un problème d'absentéisme.

Cette clarification conceptuelle étant établie, nous allons à présent nous attacher à développer les deux approches théoriques de l'absentéisme communément admises en sciences de gestion : l'approche individuelle et psychosociale (Bouville, 2009).

\section{2) Approche individuelle de l'absentéisme}

L'approche individuelle de l'absentéisme trouve son fondement dans le modèle théorique de Steers et Rhodes (1978). Ces chercheurs définissent la présence au travail comme la capacité et la motivation à être présent sur son lieu de travail. Au contraire, l'absentéisme traduirait, d'une part, l'incapacité à être présent (problèmes familiaux, problèmes de transports, maladie, etc.) et, d'autre part, l'absence de motivation au travail influencée par des attitudes liées au travail (faible satisfaction dans son emploi ou son travail en général, ou un faible degré d'implication organisationnelle).

A partir des travaux fondateurs de Steers et Rhodes (1978), plusieurs modèles de causalité ont examiné le lien entre l'absentéisme et, respectivement, la satisfaction au travail et l'implication organisationnelle (Brooke, 1986; Brooke \& Price, 1989; Gellatly, 1995; Hanisch \& Hulin, 1991 ; Meyer, 1997). Si ces deux derniers concepts sont fortement corrélés, ils sont empiriquement distincts (Brooke et al., 1988). En effet, la satisfaction au travail est une attitude positive à l'égard de son travail, influencée par la perception générale qu'à un employé de son travail et de ses caractéristiques (Meysonnier \& Roger, 2006). L'implication organisationnelle $(\mathrm{IO})$ est, elle, le lien psychologique qui unit un employé à son organisation. Dans ce sens, Allen et Meyer (1990) identifient trois composantes de l'IO : affective, normative et calculée. L'implication organisationnelle affective (IOA) reflète le désir qu'à un salarié de rester membre de son organisation. Elle traduit l'identification et l'attachement émotionnel d'un salarié à son organisation. L'implication organisationnelle normative (ION) reflète le devoir que ressent un salarié de rester membre de son organisation. Elle traduit l'obligation morale que ressent un salarié de demeurer au sein de son organisation. Enfin, l'implication organisationnelle calculée (IOC) reflète l'obligation que ressent un salarié de rester membre de son organisation. Cette forme d'implication se développe lorsque qu'un salarié se rend compte des coûts associés à son départ de l'organisation ou lorsque ses opportunités d'emploi sont limitées. C'est dans cette perspective que l'IOC est perçue comme une composante négative de l'IO (Woods et al., 2012).

A l'appui de ces définitions, l'absentéisme est appréhendé comme le reflet d'attitudes « invisibles » liées au travail (Hanisch \& Hulin, 1991) : c'est une réponse comportementale à l'insatisfaction au travail ou une faible implication organisationnelle (Gellatly, 1995). Suivant cette logique, un employé satisfait ou impliqué à l'égard de son organisation serait moins susceptible de s'absenter, car il serait davantage motivé à agir dans le sens des objectifs et

\footnotetext{
3 ADRH GCT (2016), L'absentéisme dans les collectivités territoriales : mesures et pratiques, Dossier de Presse.
} 
attentes de son organisation (Blau et Boal, 1987 ; Steers et Rhodes, 1978). Les données empiriques n'ont donné, toutefois, que des résultats mitigés quant à ces hypothèses (Hackett, 1989 ; Mayer \& Schoorman, 1992 ; Randall, 1990 ; Harrison et al., 2006 ; Mathieu et Zajac, 1990), montrant la nécessité de continuer à sonder les relations entre ces construits.

Ainsi, il n'existe pas d'unanimité en ce qui concerne la relation entre la satisfaction au travail et l'absentéisme, que cette relation soit directe ou indirecte. Alors que Kaiser (1998) note une corrélation significative et négative entre la satisfaction au travail et l'absentéisme, la plupart des travaux académiques concluent que la satisfaction au travail n'est pas un prédicteur pertinent de l'absentéisme (Brooke et Price, 1989). Dès lors, satisfaction au travail n'est plus en lien causal direct mais indirect avec l'absentéisme (Hackett, 1989). Les travaux de Brooke (1986 ; Brooke \& Price, 1989) montrent ainsi que la satisfaction au travail médiatise les effets des facteurs organisationnels (rémunération, tension de rôle, perception de justice) sur l'absentéisme. Mais ces résultats sont infirmés par Golberg et Waldman (2000).

De manière spécifique, quand au lien entre l'IO et l'absentéisme, les données empiriques sont tout aussi contradictoires, allant d'une absence totale d'effet entre ces deux construits (Brooke \& Price, 1989) à des relations modestes (Hackett, 1989; Harrison et al., 2006 ; Mayer \& Schoorman, 1992; Randall, 1990). Lorsque les chercheurs ont tenu compte des trois composantes de l'IO, les résultats de leur recherche sont tout aussi discordants. Ainsi, Falkenburg et Schnys (2007) ne trouvent aucune relation significative entre l'absentéisme et les trois composantes de l'IO. En revanche, l'IOA apparaît comme un prédicteur pertinent de l'absentéisme volontaire ou de la fréquence de l'absentéisme : la relation entre ces deux construits est significative et négative (Allen \& Meyer, 1996 ; Burton et al., 2002 ; Meyer et al., 1993, 2002 ; Somers, 1995; Woods et al., 2012). Les relations entre l'IOC et l'ION, respectivement, et l'absentéisme sont, elles, plus mitigées. Alors que certaines études empiriques montrent des liens significatifs et négatifs entre l'ION et l'absentéisme volontaire (Burton et al., 2002 ; Meyer, 1997 ; Hackett et al., 1994 ; Woods et al., 2012), d'autres ne trouvent aucune relation entre ces deux construits (Somers, 1995). De manière générale, aucun lien significatif n'a été trouvé entre l'IOC et l'absentéisme (Allen et Meyer, 1996; Meyer et al., 1993 ; Somers, 1995). Woods et al. (2012) font exception en la matière en montrant que l'IOC est liée positivement à l'absentéisme.

La variation de ces corrélations tiendrait à trois raisons. D'abord, à une mauvaise mesure de l'absentéisme, les méthodes spécifiques usitées pour mesurer ce phénomène (fréquence vs durée, absence volontaire vs involontaire) pouvant influencer l'intensité de ses relations avec l'IO et la satisfaction au travail (Blau et Boal, 1987 ; Sagie, 1998). Ensuite, à une absence de prise en considération des trois composantes de l'IO (Blau et Boal, 1987 ; Mathieu et Zajac, 1990), alors que Meyer et Allen (1991) notent que chacune de ses composantes peut exercer des effets indépendants sur un comportement en particulier. D'où la nécessité d'étudier l'impact spécifique des trois composantes de l'IO sur l'absentéisme. Enfin, l'incohérence des données empiriques tiendrait à des effets d'interaction entre la satisfaction au travail et l'IO (Sagie, 1998). Au global, peu de recherches se sont attachées à comprendre l'absentéisme par le biais des effets combinés de la satisfaction au travail et de l'IO (Sagie, 1998 ; Falkenburg et Schnys, 2007). Dans cette veine, les auteurs suggèrent que la relation négative de ces deux attitudes combinées sur l'absentéisme devrait produire une relation positive (Sagie, 1998 ; Falkenburg et Schnys, 2007). La satisfaction au travail aurait donc un effet exacerbant sur la relation entre l'IO et l'absentéisme : les personnes ayant une forte IOA tendraient à avoir un niveau d'absentéisme plus élevé quand leur niveau de satisfaction au travail est élevé. Toutefois, toute chose égale par ailleurs, le niveau d'absentéisme d'une personne fortement impliquée envers son organisation et satisfaite de son travail sera plus faible que celui d'une 
personne moins impliquée et moins satisfaite. L'ensemble de ces observations nous amène à formuler les hypothèses suivantes :

H1. L'IOA et l'ION sont liées négativement à l'absentéisme

H2. L'IOC est liée positivement à l'absentéisme

H3. La satisfaction au travail est liée négativement à l'absentéisme

H4. La satisfaction au travail modère positivement la relation entre l'IO et l'absentéisme

H4.a. La relation entre l'IOA et l'absentéisme est modérée positivement par la satisfaction au travail: les personnes ayant une forte IOA tendraient à avoir un niveau d'absentéisme plus élevé quand leur niveau de satisfaction au travail est élevé, comparativement à leurs homologues ayant un niveau de satisfaction plus faible.

H4.b. La relation entre l'ION et l'absentéisme est modérée positivement par la satisfaction au travail: les personnes ayant une forte ION tendraient à avoir un niveau d'absentéisme plus élevé quand leur niveau de satisfaction au travail est élevé, comparativement à leurs homologues ayant un niveau de satisfaction plus faible.

H2.c. La relation entre l'IOC et l'absentéisme est modérée positivement par la satisfaction au travail: les personnes ayant une forte IOC tendraient à avoir un niveau d'absentéisme plus élevé quand leur niveau de satisfaction au travail est élevé, comparativement à leurs homologues ayant un niveau de satisfaction plus faible.

Au-delà d'être un comportement individuel, décrit ici, l'absentéisme s'inscrit aussi dans une dynamique sociale (Nicholson et al., 1977). Ce point de vue est défendu par l'approche psychosociale de l'absentéisme.

\section{3) Approche psychosociale de l'absentéisme}

Selon l'approche psychosociale, le contexte social dans lequel un individu est amené à exercer son activité de travail influence sa décision de s'absenter ou non. Deux modèles de l'absentéisme vont étayer cette perception : le modèle de la théorie sociale de l'absentéisme et le modèle des exigences et des ressources du travail.

Le modèle de la théorie sociale de l'absentéisme (Chadwick-Jones et al., 1982) envisage l'absentéisme comme le produit des rapports sociaux qu'entretiennent les travailleurs au sein d'un groupe ou avec leur employeur. La qualité des différentes interactions sociales, de même que le respect des obligations mutuelles qui unit l'employé à son employeur dans le cadre d'un contrat psychologique, conditionnent ainsi le comportement d'absence des employés (Nicholson et Johns, 1985).

Le modèle des exigences et des ressources du travail (Demerouti et al., 2001a et b) permet d'expliquer l'absentéisme par le biais de deux processus, semblables à ceux énoncés par Steers et Rhodes (1978). D'une part, un processus de détérioration de la santé, affectant la capacité à être présent. Il se caractérise par des exigences persistantes de travail (tension de rôle, charge de travail, etc.) qui affaiblissent les ressources du travail (autonomie, soutien social, etc.) et conduisent à l'épuisement professionnel. Bakker et al. (2003) montrent alors que l'épuisement professionnel modère positivement la relation entre les exigences de travail et la durée de l'absentéisme. D'autre part, un processus stimulant, qui contribue à la motivation à être présent. Dans ce cas, la disponibilité des ressources du travail affaiblit les effets négatifs des exigences de travail et conduit à l'implication organisationnelle. Les travaux de Bakker et al. (2003) soulignent ici que l'implication organisationnelle modère négativement la relation entre les ressources du travail et la fréquence des absences : en effet 
lorsque les ressources du travail sont élevées, les personnes ayant une forte implication organisationnelle réduisent la fréquence de leur absence, comparativement à leurs homologues moins impliqués. Schaufeli et Bakker (2004) concluront alors que ce second processus, qualifié de stimulant, équivaut à un processus de démotivation au travail aidant à comprendre les comportements de désengagement et de retrait au travail à travers l'absence des ressources du travail. Suivant cette logique, la relation entre le contexte social et l'absentéisme serait conditionnée par les attitudes au travail et l'état de santé des employés (Bakker et Demerouti, 2007).

Pour notre étude, nous avons fait le choix de nous intéresser à une variable de ce contexte social, touchant tant la théorie sociale de l'absence que le modèle des exigences-ressources du travail : le soutien organisationnel perçu (SOP). Ce choix s'explique, d'une part, par la faiblesse des données empiriques sur le lien entre le SOP et l'absentéisme, nécessitant des investigations plus importantes en la matière D'autre part, par la pertinence de ce concept dans le contexte de mutation que traversent les collectivités territoriales et la déstabilisation sociale qui l'entoure. En effet, le SOP traduit l'évaluation faite par un salarié du degré d'implication de l'organisation à son égard (Eisenberger et al., 1986). Il est défini comme « la manière dont le salarié estime que son organisation prend en compte ses efforts, valorise son investissement personnel et contribue à son bien-être professionnel, au travers de décisions et de politiques de ressources humaines, qui favorisent la qualité de son environnement de travail » (Paillé, 2007 : 350). Trouvant son origine dans la théorie de l'échange social (Blau, 1964), qui stipule que les comportements et attitudes favorables à la performance individuelle sont expliqués par les différentes relations d'échange qui se réalisent au sein des organisations, le SOP est étroitement lié à la norme de réciprocité : ce n'est qu'après avoir évalué ce qu'il a reçu de son organisation que le salarié se sentira obligé ou non d'adopter un certain nombre d'attitudes ou comportements performants (Coyle-Shapiro et al., 2004). Ici, la relation d'échange est envisagée en termes de ressources matérielles (condition de travail, communication appropriée, formation, rémunération, carrière, etc.) et non-matérielles (écoute, soutien, qualité des échanges leader-member, reconnaissance de la contribution et souci du bien-être des salariés, etc.), octroyées par l'organisation à ses salariés. Dans cette veine, plus un salarié perçoit que son organisation contribue de manière significative à sa qualité de vie au travail en lui offrant les ressources qu'il valorise, tant pour son bien-être personnel que pour l'aider à mener à bien son travail, plus il sera enclin à l'aider à son tour en adoptant des attitudes et comportements organisationnels performants contribuant à l'efficacité organisationnelle (Eisenberger et al., 2001 ; Rhoades et al., 2001 ; Rhoades \& Eisenberger, 2002). Le SOP contribuerait ainsi à réduire l'absentéisme (Cunningham et MacGregor, 2000 ; Eisenberger et al., 1986 ; 1990). L'ensemble de ces observations nous conduit ainsi à formuler les hypothèses suivantes :

H5 : Le SOP est lié négativement à l'absentéisme.

H6. La relation entre le SOP et l'absentéisme est modérée négativement par la satisfaction au travail : les personnes ayant une forte perception du soutien organisationnel tendraient à avoir un niveau d'absentéisme plus faible quand leur niveau de satisfaction au travail est élevé, comparativement à leurs homologues ayant un niveau de satisfaction plus faible.

$\mathrm{Au}$ regard des approches théoriques de l'absentéisme mobilisées, ce phénomène est appréhendé au total en sciences de gestion comme un comportement de retrait lié aux transformations de l'organisation du travail et des conditions de travail au cours des dernières décennies (Johns, 1997 ; 2001), expliqué par des facteurs attitudinaux (Gellatly, 1995 ; Steers $\&$ Rhodes, 1978) et reflétant un dysfonctionnement organisationnel (Weiss, 1979 ; Giraud, 1987 ; Martocchio \& Jimeno, 2003). Ainsi, un manque de soutien, un faible niveau d'équité perçu ou d'implication, ou encore la dégradation des relations sociales sont des facteurs 
influençant l'accroissement de l'absentéisme (Eriksen et al., 2004; Josephson et al., 2008). En ce sens, l'absentéisme traduirait un attachement négatif, une désaffection envers son organisation (Hanisch \& Hulin, 1991), "un renoncement à l'engagement d'être présent de manière assidue à son poste" (Bouville, 2009: 7), une conduite d'évitement vis-à-vis d'aspect négatifs du travail (Bakker et al., 2003 ; Chadwick-Jones et al., 1982 ; Johns, 1997 ; Shapira-Lishchinsky \& Rosenblatt, 2009).

\section{2) Methodologie et Resultats}

Notre méthodologie et résultats sont présentés à travers plusieurs points. Dans un premier temps, nous abordons le recueil des données et les caractéristiques de notre échantillon. Dans un second temps, nous présentons nos instruments de mesures et méthodes d'analyses. Dans un troisième temps, nous présentons nos résultats.

\section{1) Recueil des données et caractéristiques de l'échantillon}

L'étude empirique a été réalisée en novembre 2017 dans le cadre d'une recherche plus importante sur la situation professionnelle des agents territoriaux et à l'appui d'un questionnaire hébergé en ligne (après nettoyage des données, $\mathrm{N}=924$ ). L'échantillon se compose de $37,8 \%$ d'hommes contre $62.2 \%$ de femmes. Près de $15.8 \%$ des répondants ont moins de 35 ans, $30 \%$ ont entre 35 et 44 ans et 54,2\% ont plus de 45 ans. Les répondants ayant un statut de fonctionnaire représentent $88.9 \%$ de notre échantillon, contre $11.1 \%$ de non-titulaire. $90,6 \%$ d'entre eux déclarent travailler à temps plein et $57,9 \%$ dans la filière administrative. Ils sont $66.5 \%$ à exercer dans une commune et $17,9 \%$ dans une intercommunalité. Enfin, la majorité des répondants travaillent dans des collectivités de grande taille: $61.9 \%$ des répondants exercent dans des collectivités d'au moins 50000 habitants, $16.5 \%$ dans des collectivités de 20000 à 49999 habitants.

\section{2) Instruments de mesures et procédures}

L'absentéisme est mesuré par le biais de deux indicateurs : la fréquence et la durée des absences. La fréquence des absences dénombre le nombre d'épisodes d'absence-maladie, hors congé maternité, sur une période cible relative aux 12 derniers mois. Cinq modalités de réponses ont été proposées aux répondants allant de $1=$ «Pas d'arrêt » à $5=$ « 6 arrêts et plus ». La durée des absences dénombre le nombre de jours d'absence-maladie, hors congé maternité, sur une période cible relative aux 12 derniers mois. Cinq modalités de réponse ont été proposées aux répondants allant de $1=« 0$ jour $»$ à $5=$ «30 jours et plus ». Nous mesurons ici un absentéisme global, sans distinction du caractère volontaire ou involontaire de l'absentéisme, pour les raisons évoquées précédemment. Les données sur l'absentéisme ont été obtenues sur la base de l'auto-déclaration (Johns, 1994 ; Sagie, 1998).

Le SOP est mesuré par la version courte de l'échelle d'Eisenberger et al. (1986), proposée par Quenneville, Bentein et Simard (2010), contenant 5 items (alpha de Cronbach $=0.91)$. L'IO a été mesurée à l'aide de la version réduite de l'échelle de Allen et Meyer (1996), proposée en version française par Belghiti-Mahut et Briole (2004). Composée de 18 items (6 items pour chaque composante), elle présente l'avantage d'avoir une meilleure capacité à discriminer les 3 composantes et présentent des propriétés psychométriques satisfaisantes. La satisfaction au travail (SAT) est, elle, mesurée par 4 items de l'échelle (Amar ???.) Ces trois variables ont été évaluées avec une échelle de Likert à cinq points, allant de $1=$ «Pas du tout d'accord », à $5=$ " Tout à fait d'accord ». L'ensemble des items a été adapté au contexte des collectivités territoriales. 
Après vérification de la qualité des données brutes, les items ayant des valeurs manquantes supérieures au seuil de 10\% ont été supprimés. La pertinence et l'affectation des énoncés de chaque construit de l'étude ont été sondées par le biais d'une analyse factorielle exploratoire (méthode d'extraction: ACP, méthode de rotation: Varimax). Suite à ces analyses préliminaires des données empiriques, l'échelle à du SOP a été réduite à 4 items (exemple : ma collectivité se soucie réellement de mon bien-être; ma collectivité valorise ma contribution à son bon fonctionnement). L'échelle de l'IO, dont la structure à trois composantes à été confirmée par l'AFE, a été réduite à 10 items : 4 items pour la mesure de 1'IOA (exemple: je me sens membre à part entière de cette collectivité), 4 items pour la mesure de l'ION (exemple: je ne quitterais pas cette collectivité, car je me sens redevable envers les personnes qui y travaillent) et 2 items pour la mesure de l'IOC (exemple : je pense que je n'ai pas assez d'opportunités de rechange pour quitter cette collectivité). Pour cette dernière mesure, les deux items maintenus sont relatifs à une opportunité d'emploi limitée. Enfin, l'échelle de la SAT a été réduite à 3 items (exemple : je suis satisfait du sentiment d'accomplissement que je retire de mon travail). L'Analyse factorielle confirmatoire fait apparaître une très bonne cohérence interne des construits résultant de la phase d'épuration (voir infra le tableau présentant les alphas de Cronbach de chaque construit entre parenthèse). Enfin, le test de colinéarité entre les variables de l'étude, présenté infra, nous permet de conclure à une absence de risque de multicolinéarité. L'ensemble de ces constations nous autorise à entamer le processus d'analyse de nos données

\section{3) Résultats}

Les statistiques descriptives et les corrélations entre les variables de l'étude figurent au tableau 1. Une attention particulière doit, tout d'abord, être accordée à l'analyse des statistiques descriptives. En effet, les moyennes arithmétiques font apparaître un niveau de soutien organisationnel, de satisfaction au travail, d'implication organisationnelle affective et calculée, supérieur à la moyenne théorique, qui est de 3 pour une échelle de Lickert à 5 points. A l'inverse les moyennes de l'absentéisme et de l'ION sont inférieures à la moyenne théorique. Ce qui pourrait nous amener, de manière erronée, à conclure, d'une part, que les répondants percevraient la présence d'un soutien organisationnel ou encore qu'ils seraient globalement satisfaits au travail et impliqués envers leur organisation de manière affective et calculée. D'autre part, qu'ils ne seraient pas impliqués de manière normative envers leur organisation et qu'ils manifesteraient un absentéisme quasi-nul. Or, si nous comparons la valeur des moyennes arithmétiques de chaque variable à celle de leur médiane, qui est une mesure moins affectée par les valeurs extrêmes, nous nous apercevons que pour le SOP, l'IOA, l'ION et la satisfaction au travail, la valeur de leur moyenne arithmétique est inférieure à celle de leur médiane. L'inverse est constaté pour l'absentéisme et l'IOC. Ce qui nous amène aux conclusions suivantes : les agents territoriaux auraient une perception dégradée du SOP, ainsi qu'un faible niveau de satisfaction au travail, d'implication organisationnelle affective et normative. En revanche, ils manifestent une implication organisationnelle calculée. Pour ce qui est de l'absentéisme, la moyenne arithmétique étant proche de 2, nous sommes en présence d'un absentéisme ponctuel, caractérisé par des absences-maladie de courte durée et de fréquence faible (Bouville, 2009). Je ne pense pas que tu puisses faire une moyenne sur l'absentéisme car les valeurs mobilisées n'indiquent pas une échelle d'importance, mais une typologie...

Par ailleurs, des relations significatives et négatives apparaissent entre l'absentéisme et, respectivement, le SOP, l'IOA et la satisfaction au travail. Ainsi, plus les personnes perçoivent une implication de leur organisation à leur égard, plus elles sont satisfaites au travail ou impliquées affectivement envers leur organisation, moins elles auront tendance à 
présenter de 1'absentéisme. Les hypothèses H3 et H5 sont dès lors validées. Au contraire, l'absentéisme et l'IOC sont corrélés significativement et positivement: ainsi, plus les personnes sont impliquées de manière calculée envers leur organisation, plus elles sont susceptibles de manifester de l'absentéisme. L'hypothèse $\mathrm{H} 2$ est donc validée. Par contre, aucune relation n'a été trouvée entre l'ION et l'absentéisme. L'hypothèse 1 est donc partiellement validée.

Tableau 1. Statistiques descriptives et corrélations entre les variables de l'étude

\begin{tabular}{|c|c|c|c|c|c|c|c|c|}
\hline Variable du modèle & Moyenne & Ecart-type & 1 & 2 & 3 & 4 & 5 & 6 \\
\hline 1. $\mathrm{ABS}$ & 1,71 & ,91356 & $(, 827)$ & & & & & \\
\hline 2. SOP & 2,87 & 977 &,$- 149^{* *}$ & $(, 920)$ & & & & \\
\hline 3. IOA & 3,36 & ,798 &,$- 093^{*}$ &, $371^{* *}$ & $(, 738)$ & & & \\
\hline 4. IOC & 3,14 & 1,05 &, $125^{* *}$ &,$- 290^{* *}$ &,$- 157^{* * *}$ & $(, 836)$ & & \\
\hline 5. ION & 2,44 & ,782 &,- 034 &, $325^{* *}$ &, $376^{* * *}$ &,$- 121^{* *}$ & $(, 736)$ & \\
\hline 6. SAT & 3,80 & ,867 &,$- 113^{* * *}$ &, $430^{* *}$ &, $303^{* *}$ &,$- 215^{* *}$ &, $210^{* *}$ & $(, 851)$ \\
\hline
\end{tabular}

Les résultats de la régression multiple confirment le sens des corrélations significatives trouvées précédemment. Toutefois, ils mettent en évidence que seuls le SOP et l'IOC (ION non? en gras plus bas) apparaissent comme des variables prédictives pertinentes de l'absentéisme dans la fonction publique territoriale.

Tableau 2. Résultats de la régression multiple

\begin{tabular}{|c|c|c|c|c|c|c|}
\hline \hline & \multirow{2}{*}{ Variables du modèle } & $\boldsymbol{\beta}$ & $\boldsymbol{p}$ & \multicolumn{2}{|c|}{ IC à 95\% pour B } & \multicolumn{2}{c|}{ Statistiques de colinéarité } \\
\cline { 4 - 7 } & & Borne inférieure & Borne supérieure & Tolérance & VIF \\
\hline \hline SOP &,- 100 & $\mathbf{, 0 2 3}$ &,- 173 &,- 013 &, 695 & 1,440 \\
IOA &,- 042 &, 315 &,- 141 &, 045 &, 770 & 1,299 \\
ION &, 084 &, $\mathbf{0 2 9}$ &, 007 &, 138 &, 904 & 1,106 \\
IOC &, 035 &, 393 &,- 052 &, 132 &, 817 & 1,223 \\
SAT &,- 047 &, 255 &,- 134 &, 036 &, 783 & 1,278 \\
\hline
\end{tabular}

$N=732$, toute observation incomplète

Afin de tester les hypothèses de modération de la satisfaction au travail, nous avons procédé à une analyse de régression modérée à l'appui de la macro process de Hayes (2013). A cet effet, les moyennes ont été centrées. La méthode utilisée est celle du boostrapp (=10 000), avec un intervalle de confiance à $95 \%$, une significativité à $\mathrm{p}<0.05$. Les effets conditionnels ont été évalués aux percentiles 16,50 et 84 , afin de garder la certitude de s'inscrire dans le domaine des valeurs plausibles pour le modérateur (Hayes, 2013). En outre, la technique de JohnsonNeyman a été utilisée dans le but de déterminer la valeur précise du modérateur à partir de laquelle l'effet conditionnel des variables sondées sur l'absentéisme est significatif.

Les résultats de cette analyse (tableau 3) montrent que le SOP a un effet direct et négatif sur l'absentéisme (Modèle 1) : le pouvoir explicatif du modèle reste inchangé, mais perd sa significativité $\left(\mathrm{R}^{2}=0.024 ; \mathrm{p}<0.01\right)$ lorsque l'interaction entre le SOP et la satisfaction au travail est introduite. L'hypothèse H6 est donc non validée. De même, l'IOC et la satisfaction au travail possèdent respectivement un effet direct positif et négatif sur l'absentéisme (Modèle 
3) : le pouvoir explicatif du modèle se dégrade avec un $\mathrm{R}^{2}$ passant de $0.024(\mathrm{p}<0.01)$ à 0.023 , lorsque l'interaction entre l'IOC et la satisfaction au travail est introduite. L'hypothèse H4.c est donc non validée Toutefois, l'effet de l'IOC sur l'absentéisme est légèrement plus puissant que celui de la satisfaction au travail $(B=0.0925$ vs $B=0.0907)$. Ainsi mis en relation avec l'IOC, la satisfaction au travail apparaît comme une condition nécessaire, mais insuffisante pour baisser le niveau d'absentéisme. Le Modèle 4 montre qu'aucun effet direct de l'ION sur l'absentéisme n'a été trouvé. Il en va de même pour l'effet modérateur de la satisfaction au travail sur la relation entre l'ION et l'absentéisme. Toutefois, dans ce modèle la satisfaction au travail possède un effet direct et négatif sur l'absentéisme, avec un pouvoir explicatif faible et moins significatif comparativement au Modèle $3\left(\mathrm{R}^{2}=0.015\right.$ avec $\mathrm{p}<0.05$ vs $\mathrm{R}^{2}=0.024$ avec $\mathrm{p}<0.01$ ). L'hypothèse H4.b est donc non validée.

Tableau 3. Résultats de la régression modérée

\begin{tabular}{|c|c|c|c|c|c|c|}
\hline & $\mathbf{R}^{2}$ & $\mathbf{R}^{2}$ change & Coeff & $\mathbf{p}$ & LLCI & ULCI \\
\hline Modèle 1 &, $024 * *$ &, 0000 & & & & \\
\hline SOP & & &,- 1116 & ,0030 &,- 1851 & - ,0381 \\
\hline SAT & & &,- 0619 &, 1737 &,- 1511 &, 0273 \\
\hline SOP*SAT & & &, 0033 & ,9335 &,- 0735 &, 0800 \\
\hline Modèle 2 & ,026 &, $0093 * *$ & & & & \\
\hline IOA & & &,- 0790 & 0704 &,- 1646 &, 0066 \\
\hline SAT & & &,- 1147 &, 0050 &,- 1946 &,- 0347 \\
\hline $\mathrm{IOA} * \mathrm{SAT}$ & & &,- 1096 & ,0085 &,- 1911 &,- 0281 \\
\hline Modèle 3 &, $024 * *$ & ,0007 & & & & \\
\hline IOC & & & ,0925 &, 0045 &, 0287 & ,1562 \\
\hline SAT & & &,- 0907 &, 0227 &,- 1687 &,- 0127 \\
\hline $\mathrm{IOC}^{*} \mathrm{SAT}$ & & &,- 0243 & ,4632 &,- 0894 & 0407 \\
\hline Modèle 4 &, $015^{*}$ &, 0024 & & & & \\
\hline ION & & &,- 0079 &, 8562 &,- 0936 & ,0778 \\
\hline SAT & & &,- 1301 & ,0014 &,- 2098 &,- 0503 \\
\hline ION*SAT & & &,- 0632 &, 1811 &,- 1559 &, 0295 \\
\hline
\end{tabular}

Seule la relation entre l'IOA et l'absentéisme est modérée par la satisfaction au travail (Modèle 2). En effet, le pouvoir explicatif du modèle se trouve amélioré lorsque l'interaction entre l'IOA et la satisfaction au travail est introduite, avec un $\mathrm{R}^{2}$ passant de 0.016 à 0.026 ( $\mathrm{p}<$ 0.01). La corrélatio,, significative et positive entre l'IOA et la satisfaction au travail (tableau 1), n'invalide pas l'effet interactif trouvé entre ces deux attitudes sur l'absentéisme, mais accorde davantage de confiance à la conclusion que cet effet est réel (Mathieu et Kohler, 1990). Plus spécifiquement, l'effet modérateur de la satisfaction au travail sur la relation entre l'IOA et l'absentéisme devient significatif pour une valeur de la satisfaction au travail équivalente à $0.063(\mathrm{p}<0.05)$. La technique de Johnson-Neyman montre que $62.4 \%$ des personnes de notre échantillon se situe au-dessus de cette valeur, confirmant ainsi que la relation entre l'IOA et l'absentéisme est bien modérée par la satisfaction au travail. En outre, nous pouvons constater que la satisfaction au travail modère négativement la relation entre l'IOA et l'absentéisme. Cette relation est représentée dans la figure 1 qui montre que :

(1) le niveau d'absentéisme est plus faible pour les personnes ayant un faible niveau d'IOA associé à un niveau élevé de satisfaction au travail, comparativement à leurs homologues moins satisfaits 
(2) le niveau d'absentéisme est significativement plus faible pour les personnes ayant un fort niveau d'IOA associé à un niveau élevé de satisfaction au travail, comparativement à leurs homologues moins satisfaits ;

(3) le niveau d'absentéisme baisse de manière importante pour les personnes ayant un fort niveau d'IOA associé à un niveau élevé de satisfaction au travail, comparativement à leurs homologues moins impliqués affectivement envers leur organisation.

(4) Le niveau d'absentéisme est légèrement plus élevé (1.83 vs 1.87) pour les personnes ayant un haut niveau d'IOA associé à un faible niveau de satisfaction au travail, comparativement à leurs homologues moins impliqués.

En conséquence, l'hypothèse H4.a est rejetée, les résultats de la recherche mettant en évidence que le niveau d'absentéisme baisse considérablement lorsqu'un fort niveau IOA est associé à un fort niveau de satisfaction au travail. Mise en lien avec l'IOA, la satisfaction au travail apparaît alors comme une condition nécessaire et indispensable pour baisser le niveau d'absentéisme.

Figure 1. Effet de la satisfaction au travail sur la relation entre l'implication organisationnelle affective et l'absentéisme

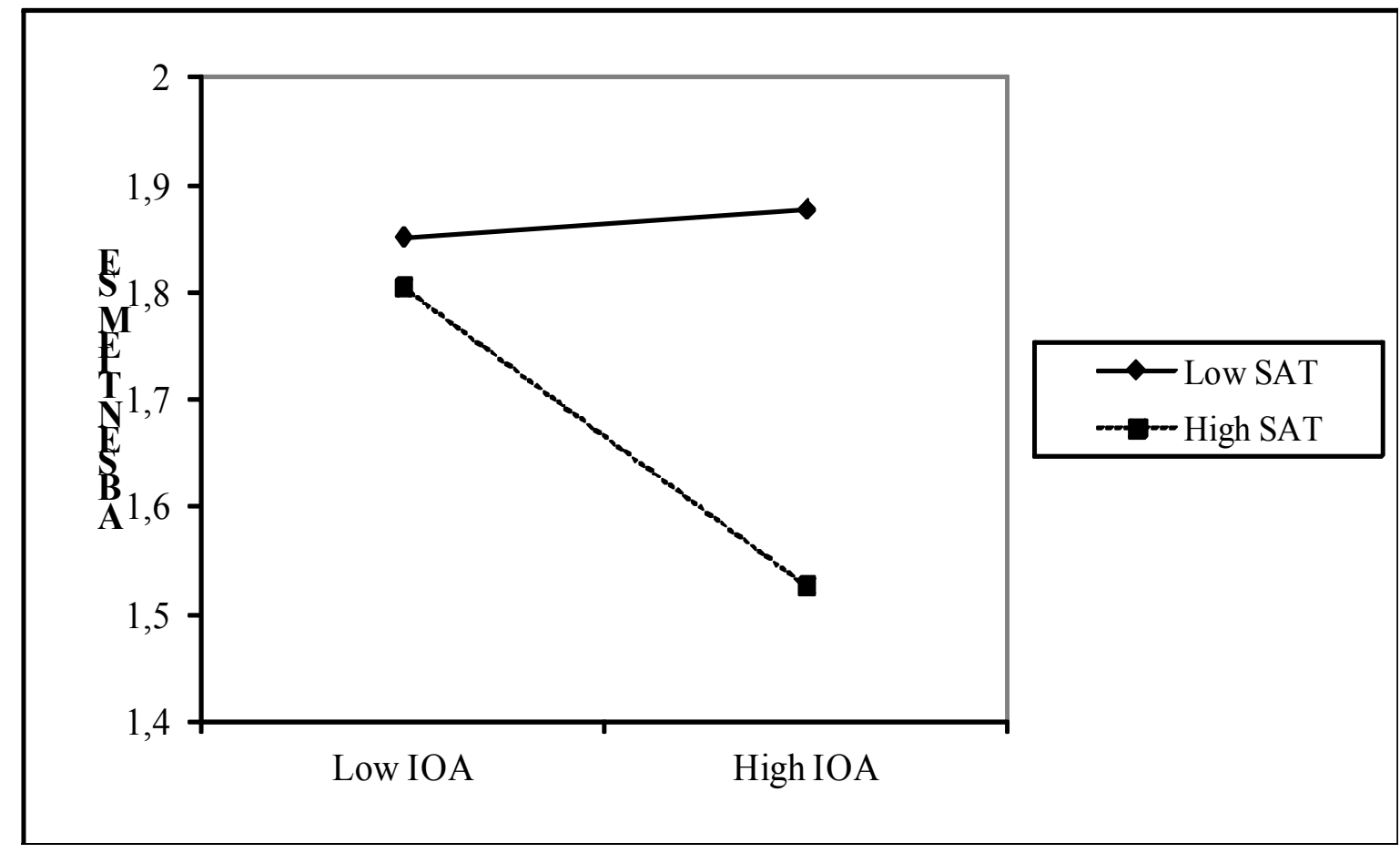

Les résultats étant présentés, nous les discutions à présent au regard de la littérature mobilisée.

\section{3) Discussion}

Notre discussion est présentée à travers plusieurs points. Dans un premier temps, nous abordons les apports théoriques et managériaux, ainsi que nos pistes de recherche. Dans un second temps, nous présentons les limites et prolongements de cette recherche.

\section{1) Apports théoriques, managériaux et pistes de recherche}

Bien que de nombreuses recherches se soient attachées à comprendre les causes de l'absentéisme, il n'existe pas à ce jour d'étude empirique consacré à ce phénomène en 
contexte public local. Notre recherche apporte ainsi une première contribution à la littérature en comblant cette lacune.

Plus spécifiquement, les deux variables prédictives de l'absentéisme, dans le contexte spécifique de la fonction publique territoriale, sont le SOP et l'IOC. Les résultats de la recherche montrent que le SOP a une relation directe, significative et négative avec l'absentéisme : plus un agent territorial estime que sa collectivité contribue à la qualité de son environnement de travail, se soucie de son bien-être et valorise ses efforts, en lui procurant les ressources matérielles et immatérielles qu'il valorise, moins il manifeste de comportements contre-productifs, comme l'absentéisme. Ces résultats enrichissent la recherche en apportant un soutien empirique aux rares travaux sondant la relation entre le SOP et l'absentéisme (Cunningham et MacGregor, 2000 ; Eisenberger et al., 1986 ; 1990). Plus spécifiquement, le SOP permettrait de lutter contre l'absentéisme ponctuel qui a été repéré lors de l'analyse des statistiques descriptives de notre étude. Gallois (2009, p.21) qualifie ce type d'absentéisme comme un absentéisme désorganisant en raison des micro-dysfonctionnements qu'il crée au sein de l'organisation : non remplacement des personnes absentes en raison de la durée courte de leur absence, report de la charge de travail des « absents » sur les « présents », remise en question de la continuité et qualité du service public. Le SOP apparaît ainsi comme une ressource de travail qui, lorsqu'elle vient à manquer, peut entraîner des effets sur les comportements de retrait (Demourouti \& al., 2001a). En ce sens, il est considéré comme une ressource préservant la motivation à être présent au travail (Aselage \& Eisenberger, 2003 ; Wayne et al., 2002). En outre, plusieurs auteurs suggèrent que la perception qu'ont les travailleurs de l'implication de leur organisation à leur égard est contingente aux pratiques de GRH des managers de proximité (Thomas \& Ganster, 1995 ; Rhodes \& Eisenberger, 2002). En effet, ces derniers sont les plus à même, au regard de la relation étroite et singulière qu'ils entretiennent avec leur équipe, de leur proposer des ressources, tangibles ou non, leur permettant de négocier et gérer au mieux les changements et difficultés qu'ils rencontrent dans leur travail (Georges et al., 1993).

Face à l'ampleur des changements dans la fonction publique territoriale et au contexte de déstabilisation sociale qui l'entoure ${ }^{4}$, nos résultats apportent alors une contribution managériale importante en montrant que le SOP peut-être un levier potentiel de la lutte contre l'absentéisme dans la fonction publique territoriale. D'un point de vue pratique, ils suggèrent la nécessité de repenser le pilotage relationnel territorial : informer les agents sur les origines et enjeux du changement afin de les aider à mieux le comprendre, à clarifier leur rôle et mission, écouter et comprendre les inquiétudes, soutenir et accompagner les agents dans leur difficulté, encourager la réflexion constituent ainsi quelques-unes des évolutions à engager (Chomienne \& Pupion, 2009 ; Bartoli \& Chomienne, 2007 ; Mahe de Boislandelle \& Brute de Remur, 2009). Dans cette perspective, l'accompagnement des managers publics de proximité dans l'évolution de leur rôle (manager par le sens vs manager par les règles; manager par la cohérence, la participation et la réflexion vs manager par le cloisonnement et l'obéissance, manager par la valorisation vs manager par la contrainte et la sanction : Chomienne \& Pupion, 2009) devient une condition nécessaire à mettre en œuvre par les collectivités territoriales afin d'améliorer la qualité des interactions entre les managers de proximité et leur équipe. Cela participerait ainsi à l'amélioration de la perception qu'ont les agents territoriaux du soutien de

\footnotetext{
${ }^{4}$ Modification de la culture et des valeurs organisationnelles des agents territoriaux, de leur relation et condition d'emploi, baisse progressive de leur motivation au travail (Emery \& Giauque, 2012), ambigüité sur les valeurs et finalités du service public, incertitude sur leur perspective professionnelle, dégradation de leur condition de travail et de la qualité du management, absence de clarté sur les règles et procédures internes de fonctionnement, manque de reconnaissance hiérarchique, une perte de sens du travail (Enquête « Bien-être dans la fonction publique territoriale », 2014, 2015, 2016) : autant de maux traduisant cette déstabilisation sociale
} 
leur organisation et ainsi à une réduction de leur absentéisme (Wayne et al., 2002). Dans cette veine, les recherches futures gagneraient à étudier les effets conjoints du SOP, du soutien hiérarchique et de la qualité des relations «leader-member» sur l'absentéisme dans le contexte public territorial.

Nos résultats de recherche montrent également un effet direct, significatif et positif de l'IOC sur l'absentéisme. Ces résultats enrichissent la recherche. En effet, les travaux académiques antérieurs n'ont trouvé aucun lien entre ces deux construits (Allen \& Meyer, 1996 ; Somers, 1995), à l'exception des travaux de Woods et al. (2012). Nos résultats de recherche soutiennent ainsi ceux de ces derniers auteurs : plus une personne est impliquée de manière calculée envers son organisation, en raison de la perception des coûts associés à son départ de l'organisation ou des opportunités d'emploi limitées, plus elle s'absentera. En effet, une personne ayant une IOC forte ne possède pas d'autres choix que de rester dans son organisation. Elle se servira alors de l'absentéisme comme une solution de retrait lui permettant de supporter l'obligation de rester dans son organisation (Woods et al., 2012). Notre échelle de mesure de l'IOC se composant uniquement d'items relatifs à l'absence d'opportunités d'emplois, nos résultats de recherche suggèreraient que les opportunités d'emploi limitées dans la fonction publique territoriale agiraient comme un mécanisme incitatif à l'absentéisme. Ces résultats peuvent se comprendre à l'aune du concept d'absentéisme calculé de Johns et Nicholson (1982). Ces auteurs considèrent alors l'absentéisme calculé comme une monnaie d'échange : les employés utiliseraient de manière conséquente l'absentéisme afin de modifier le contrat psychologique implicite entre eux et leur employeur. Le concept de contrat psychologique soutient que l'employé et son organisation ont des attentes et obligations réciproques l'un envers l'autre (Rousseau \& Tijoriwala, 1998). Dans cette perspective, les possibilités d'évolution professionnelle pourraient être considérées par les agents territoriaux comme une obligation implicite de leur employeur à leur égard, ce d'autant plus dans un système bureaucratique régi par la carrière. Ainsi, les agents territoriaux ayant une implication calculée envers leur organisation utiliseraient l'absentéisme comme une «monnaie d'échange» face à une perception d'opportunités d'emplois limitées au sein de leur organisation et dans la fonction publique territoriale en générale.

Ces nouvelles observations apportent un éclairage managérial non négligeable dans la lutte contre l'absentéisme des agents territoriaux. En effet, d'un point de vue pratique, ils mettent l'accent sur un point de vigilance : la généralisation des dispositifs d'ajustement salarial au sein de la fonction publique territoriale, tels que la rémunération à la performance fondée sur le mérite, l'incitation financière à la présence ou la pénalisation des absences, auraient un effet contre-productif sur l'absentéisme des agents territoriaux (Sagie, 1998). Ils dégraderaient leur attachement et loyauté envers leur organisation et renforceraient leur IOC, par une perception accrue des coûts associés au départ de leur organisation et son influence néfaste sur l'absentéisme (Falkenburg \& Schyns, 2007), sans toutefois compenser leur perception d'absence d'opportunités d'emplois. D'autres auteurs soutiennent, quant à eux, que de telles pratiques de GRH, basées sur des incitations rétributives, inciteraient au présentéisme des agents territoriaux (Huver, 2013). Défini comme «la renonciation à des jours d'absencesmaladie » (Huver, 2013), le présentéisme reflète le comportement d'un travailleur qui se rendrait sur son lieu de travail alors que son état de santé, physique ou psychique, nécessiterait une absence pour raison de maladie (Johns, 2010). Dans cette veine, le présentéisme dégraderait la performance des agents dans leur tâche, ainsi que de la qualité du travail effectué et leur santé au travail sur le long terme (Gosselin \& Lauzier, 2011 ; Huver, 2013 ; Johns, 2010). Ces pratiques d'ajustement salarial censées lutter contre l'absentéisme contribueraient en ce sens à transformer l'attente sociale de présence des travailleurs sur leur 
lieu de travail en une obligation de présence (Gosselin \& Lauzier, 2011 ; Johns, 2010). Cela accentuerait alors les comportements d'absentéisme tant par la dégradation de la motivation à être présent que par celle de la capacité à être présent. Ces constations incitent ainsi les recherches futures à approfondir les relations entre l'IOC, le présentéisme, les pratiques de GRH et l'absentéisme, afin d'étayer les réflexions de la présente étude. De plus, elles suggèrent que, dans la lutte contre l'absentéisme, la fonction publique territoriale gagnerait davantage à mettre en place une pratique de GRH éthique mettant l'accent sur une meilleure anticipation des ressources humaines par une meilleure articulation entre les orientations stratégiques des collectivités et les outils/dispositifs de la GRH (formation, gestion des compétences et des carrières, etc.). Cela favoriserait en conséquence la création d'un environnement de travail positif et gratifiant, dans lequel les agents territoriaux auraient le sentiment d'être soutenus par leur organisation et pourraient s'impliquer de manière affective et normative (Woods et al., 2012 ; Bartoli et al., 2011 ; Emery et Giauque, 2012).

De plus, à l'instar des travaux antérieurs, nous avons aussi trouvé une corrélation négative et significative entre l'IOA et l'absentéisme (Allen \& Meyer, 1996; Burton et al., 2002; Meyer et al., 1993, 2002 ; Somers, 1995 ; Woods et al., 2012), mais aucune relation entre l'ION et l'absentéisme (Somers, 1995 ; Falkenbuug et Schnys, 2007). Toutefois, de manière contreintuitive et à contre-sens des travaux antérieurs, nos résultats montrent que l'IOA, comme l'ION, n'apparaît pas comme un prédicteur pertinent de l'absentéisme dans la fonction publique territoriale. Ces résultats pourraient être le signe, ainsi que le suggère l'analyse de nos statistiques descriptives, d'un essoufflement de ces deux formes d'implication organisationnelle au sein de la fonction publique territoriale, au profit de l'émergence d'une implication calculée agissant en faveur des comportements d'absentéisme. En ce sens, l'absentéisme dans la fonction publique territoriale témoignerait, d'une part, d'une "désaffection » au travail des agents territoriaux et, d'autre part, d'un absentéisme calculé reflétant des attitudes de travail négatives à l'égard de l'organisation (IOC). Ces résultats contribuent, d'un point de vue managérial, à une meilleure appréhension et caractérisation de l'absentéisme des agents territoriaux. Les futures recherches devraient alors examiner plus en détail les raisons qui poussent les agents territoriaux à exprimer moins d'attachement, de sentiment d'obligation morale et de réciprocité envers leur organisation. Cela améliorerait davantage la compréhension des influences de l'IO sur l'absentéisme dans la fonction publique territoriale. En outre, nos résultats de recherche incitent à réinvestir, dans la gestion quotidienne des agents territoriaux, le «travail de lien» inhérent au management des ressources humaines (Thévenet \& Vachette, 1992; Thévenet, 2009). Ces constatations renforcent la nécessité, déjà énoncée dans le cadre de cette discussion, d'aller dans le sens d'une nouvelle réflexion sur le pilotage relationnel des agents territoriaux, en renforçant le rôle d'accompagnement des managers de proximité.

Enfin, nos résultats de recherche montrent également que la satisfaction au travail modère négativement la relation entre l'IOA et l'absentéisme. Ainsi :

(1) Les agents territoriaux ayant un niveau élevé d'IOA et satisfaction de travail diminuent considérablement leur absentéisme, comparativement à leurs homologues moins impliqués affectivement envers leur organisation.

(2) Les agents territoriaux ayant un fort niveau d'IOA et de satisfaction au travail ont un niveau d'absentéisme plus faible que leurs homologues moins satisfaits.

(3) Les agents territoriaux ayant une faible IOA ont un niveau d'absentéisme plus faible lorsque leur niveau de satisfaction au travail est élevé, comparativement à leurs homologues moins satisfaits 
(4) Lorsque le niveau de satisfaction au travail est faible, le niveau d'absentéisme des agents territoriaux est légèrement supérieur lorsque leur niveau d'IOA est élevé, comparativement à leurs homologues moins impliqués

Ces résultats ne confirment pas les recherches antérieures mettant en évidence un effet positif de l'interaction entre la satisfaction au travail et l'IO en général, ou l'IOA plus spécifiquement, sur l'absentéisme (Sagie, 1998 ; Falkenburg \& Schnys, 2007). En revanche, ils vont dans le sens du modèle théorique de Blau et Boal (1987) sondant l'interaction entre l'engagement au travail et l'implication organisationnelle sur l'absentéisme et le turnover. Au regard des observations et conceptualisations de ses auteurs, nos résultats montreraient que les agents territoriaux ayant un niveau élevé d'IOA et de satisfaction au travail seraient plus motivés à être présents car ils auraient un attrait à la fois pour leur organisation et leur travail. En effet, leur identification et attachement envers leur collectivité les conduiraient à adopter des comportements favorables n'allant pas à l'encontre de la bonne marche de cette dernière. De même, leur satisfaction à l'égard de leur travail, et de ses caractéristiques en général, les conduirait à faire plus d'efforts dans leur travail. Ce double effort de comportements favorables à la performance organisationnelle et individuelle agirait en faveur d'une réduction de leur niveau d'absentéisme. En outre, ces agents territoriaux, qui correspondraient au profil des «stars institutionnels" (forte IOA/forte satisfaction au travail) de Blau et Boal (1987) auraient des niveaux d'absentéisme plus faible que leur collègues moins satisfaits et moins impliqués. En revanche, ils seraient plus sensibles au travail qu'ils accomplissent et au traitement que leur organisation leur réserve, de sorte qu'un accroissement de leur niveau d'absentéisme est observé soit :

- face une désillusion à l'égard de leur organisation (faible IOA/forte satisfaction au travail), marqués par un retrait envers les efforts collectifs en faveur de l'organisation, mais un maintien des efforts individuels au travail ;

- face à une insatisfaction au travail (forte IOA/faible satisfaction au travail), marqués par un maintien des efforts collectifs pour l'organisation, mais une réduction des efforts individuels dans le travail ;

- $\quad$ soit les deux (faible IOA/faible satisfaction au travail) : dans ce dernier cas, selon Blau et Boal (1987), les personnes manifesteraient un absentéisme calculé (Johns \& Nicholson, 1982). En effet, leur faible attachement et identification aux valeurs et objectifs de l'organisation les conduiraient à diminuer leur effort collectif. Ces observations soutiennent la relation significative et positive que nous avons trouvée entre l'IOC et l'absentéisme. En même temps, ces personnes sont plus attachées aux caractéristiques de leur travail, de sorte de leur insatisfaction à l'égard de ces dernières provoquerait également un retrait de leurs efforts individuels en faveur de leur travail. Ces constations vont dans le sens d'un effet interactif entre l'IOC et la satisfaction au travail. Toutefois, les résultats de notre recherche ne montrent pas d'effet modérateur de la satisfaction au travail sur la relation entre l'IOC et l'absentéisme. Ces résultats pourraient s'expliquer par le fait que l'IOA est plus fortement liée à la satisfaction au travail en général, tandis que l'IOC est davantage liée à la satisfaction extrinsèque au travail telle que des opportunités de promotion par exemple (Blau et Boal, 1987 ; Mathieu et Zajac, 1990).

$\mathrm{Au}$ regard de ces développements, nos résultats de recherche soulignent que la satisfaction au travail est une condition indispensable et nécessaire pour réduire l'absentéisme des agents territoriaux impliqués affectivement envers leur organisation. En revanche, elle est une condition insuffisante pour réduire l'absentéisme des agents territoriaux fortement impliqués de manière calculée envers leur organisation. D'un point de vue pratique, ces résultats suggèrent la mise en œuvre de pratiques et actions de GRH améliorant simultanément le 
sentiment de satisfaction au travail et l'implication organisationnelle affective des agents territoriaux. Toutefois, afin de lutter efficacement contre l'absentéisme, les managers publics devront être attentifs à ce que ces pratiques et actions de GRH ne conduisent pas parallèlement, d'une part, à accroître des attitudes de conformité aux attentes et normes de l'organisation basées sur des jugements calculés (IOC) et, d'autre part, à accroître le présentéisme des agents territoriaux. Dans le cas contraire, ces pratiques de lutte contre l'absentéisme produiraient des effets contre-productifs sur ce comportement en contribuant à le renforcer.

Si cette recherche apporte une contribution significative à la compréhension de l'absentéisme des agents territoriaux, elle n'est toutefois pas exempte de biais.

\section{2) Limites de la recherche et prolongements}

Les deux premières limites de cette recherche résident dans la mesure de l'absentéisme. En effet, les données sur l'absentéisme ont été obtenues par auto-déclaration. Cette méthode est considérée comme une mesure valable de l'absentéisme, les auteurs montrant des corrélations modérées entre l'auto-déclaration et les méthodes de recueil de données à partir des sources de l'organisation (Johns, 1994 ; Sagie, 1998). Toutefois, elle possède un biais égocentrique pouvant conduire les répondants à une sous déclaration de leur absence-maladie (Johns, 1994). Cela peut ainsi donner lieu à des données incorrectes ou incomplètes de l'absentéisme. En outre, nous avons fait le choix de mesurer l'absentéisme par le biais d'une mesure globale, sans égard des différents types d'absentéisme identifiés dans la littérature. Or, cela peut nuire à la compréhension exhaustive de l'absentéisme en affectant l'impact spécifique des déterminants de notre étude sur les différents phénomènes d'absentéisme (Bouville, 2009; Van Thiele et al., 2006). Nos recherches futures doivent ainsi s'attacher à mieux discriminer les formes de l'absentéisme afin de vérifier et approfondir les relations trouvées dans cette étude entre le SOP, les trois composantes de l'IO, la satisfaction au travail et l'absentéisme.

Une autre limite de cette recherche porte sur la mesure de la satisfaction au travail. Nous avons fait le choix de mesurer la satisfaction globale au travail. Or, la compréhension des effets directs et modérateurs de la satisfaction au travail sur l'absentéisme pourrait être améliorée en distinguant la satisfaction intrinsèque et extrinsèque au travail. Nos futures recherches doivent aussi aller dans ce sens.

Enfin, la dernière limite de notre recherche tient à la faiblesse du pouvoir explicatif des variables indépendantes de notre étude sur l'absentéisme. Cette limite s'explique par le fait que l'absentéisme est un phénomène complexe à étudier trouvant ses sources dans une multiplicité de causes, souvent en interaction (Steel, 2003 ; Blau et Boal, 1987). Notre objectif n'étant pas de proposer un modèle explicatif global de l'absentéisme, mais de comprendre les effets du SOP, de l'IO et de la satisfaction au travail sur ce phénomène. Cette limite, même si elle impacte la validité globale de cette notre recherche, semble toutefois ne pas en affecter sa pertinence. Elle permet ainsi de compléter les recherches antérieures, sur un plan scientifique, mais aussi d'influencer les nouvelles pratiques managériales à mettre en œuvre en matière de GRH dans un contexte local mouvant et contraint impactant les agents territoriaux.

\section{BIBLIOGRAPHIE}

ALLEN, N.J., \& MEYER, J.P. (1990). The measurement and the organization. Journal of Occupational Psychology, 68, 1-18 
ALLEN, N.J., \& MEYER J.P. (1996). Affective, continuance and normative commitment to the organization: An examination of construct validity. Journal of Vocational Behaviour , 49(2), 252276

ALEXANDERSON, K., (1998). Sickness absence: a review of performed studies with focused on levels of exposures and theories utilized. Scandinavian Journal of Social Medicine, 26 (4), 241-249

ASELAGE, J., \& EISENBERGER R. (2003). Perceived organizational support and psychological contracts: A theoretical integration, Journal of Organizational Behaviour, 24, 491-509

BAKKER, A.B., DEMEROUTI, E., de BOER, E., \& SCHAUFELI, W.B. (2003). Job demands and job resources as predictors of Absence duration and frequency. Journal of Vocational Behavior, 62, 341-356

BAKKER, A.B., \& DEMEROUTI, E. (2007). The job demands-resources model: State of the art. Journal of Managerial Psychology, 22 (3), 309-328.

BARTOLI, A. (2006). Le management dans les organisations publiques, Dunod

BARTOLI, A., \& CHOMIENNE, H. (2007). Le développement du management dans les services publics : évolution ou révolution ?. Les cahiers français $n^{\circ} 339$, Les services publics, La Documentation française.

BARTOLI, A., KERAMIDAS, O., LARAT, F., \& MAZOUZ, B. (2011). Vers un management public éthique et performant. Revue française d'administration publique, 140(4), 629-639.

BELGHITI-MAHUT, S., \& BRIOLE, A. (2004). L'implication organisationnelle et les femmes cadres : une interrogation autour de la validation de l'échelle de Allen et Meyer (1996). Psychologie du Travail et des Organisations, 10(2), 145-164

BLAU, P., (1964). Exchange and power in social life, New York: Wiley

BLAU, G.J., \& BOAL, K.B. (1987). Conceptualizing how job involvement and organizational commitment affect turnover and Absenteeism. Academy of Management Review, 12(2), 288-300

BROOKE, P. (1986). Beyond the Steers and Rhodes model of employee attendance. Academy of Management Review, 11(2), 345-362.

BROOKE, P.P., RUSSELL, D.W., \& PRICE, J.L. (1988). Discriminant validation of measures of job satisfaction, job involvement, and organizational commitment. Journal of Applied Psychology, 73 (2), 139-145.

BROOKE, P.B., \& PRICE, J.L. (1989). The determinants of employee absenteeism: An empirical test of a causal model. Journal of Occupational Psychology, 62 (1), 1-19

BOUCHIKHI, H., \& KIMBERLY, J. (1992). Le management bureauganique ou comment réconcilier le clan et la bureaucratie, Gérer et comprendre, 29, 4-16

BOUVILLE, G. (2009). L'influence de l'organisation et des conditions de travail sur l'absentéisme. Analyse quantitative et étude de cas. Thèse de Doctorat en Sciences de Gestion, Université de Rennes 1

BROUWERS, I., CORNET, A., GUTIERREZ, L., PICHAUlT, F., ROUSSEAU, A., \& WARNOTTE, G. (1997). Management Humain et contexte de changement. Pour une approche constructiviste, De Boeck

BURTON, J.P., LEE, T.W., \& HOLTOM, B.C. (2002). The influence of motivation to attend, ability to attend, and organizational commitment on different types of absence behaviours. Journal of Managerial Issues, 14(2), 181-197

CHADWICK-JONES, J., NICHOLSON, N., \& BROWN, C. (1982). Social psychology of absenteeism. New York: Praeger

CHOMIENNE, H., \& PUPION, P.C. (2009). Autonomie et responsabilité des cadres publics : une mutation managériale, CNDP. 
CHATTERJI, M., \& TILLEY, C.J. (2002). Sickness, absenteeism, presenteeism, and sick pay. Oxford Economic Papers, 54(4), 669-687

COYLE-SHAPIRO, J.A.M., KESSLER, I., \& PURCELL J. (2004). Exploring organizationally directed citizenship behaviour : reciprocity or It's my job ?. Journal of Management Studies, 41, $85-106$

CUNNINGHAM, J.B., \& MCGREGOR, J. (2000). Trust and the design of work: Complementary constructs in satisfaction and performance. Human Relations, 52, 1575-1591

DALAL, R.S. (2005). A meta-analysis of the relationship between organizational citizenship behavior and counterproductive work behavior. Journal of Applied Psychology, 90 (6), 1241-1255

DAMANPOUR, F., \& SCHNEIDER, M. (2006). Phases of the adoption of innovation in organizations: Effects of environment, organization and top managers. British Journal of Management, 17, 215-236.

DAMANPOUR, F., \& SCHNEIDER, M. (2008). Characteristics of innovation and innovation adoption in public organizations: Assessing the role of managers. Journal of Public Administration Research and Theory, 19 (3), 495-522.

DELERY, J.E. (1998). Issues of fit in strategic human resource management: implications for research. Human Resource Management Review, 8, 289-309.

DEMEROUTI, E., BAKKER, A.B., de JONGE, J., JANSSEN, P.P.M., \& SCHAUFELI, W.B. (2001a). Burnout and engagement at work as a function of demands and control. Scandinavian Journal of Work and Environment and Health, 27, 279-286.

DEMEROUTI, E., BAKKER, A.B., NACHREINER, F., \& SCHAUFELI, W.B. (2001b). The job demands-resources model of burnout. Journal of Applied Psychology, 86 (3), 499-512.

EDGAR, F., \& GEARE, A. (2005). HRM practice and employee attitudes: different measures different results, Personnel Review, 5, 534-549.

EISENBERGER, R., HUNTINGTON, R., HUTCHINSON, S., \& SOWA, D. (1986). Perceived organizational support. Journal of Applied Psychology, 75(1), 51-59

EISENBERGER, R., FASOLO, M., \& DAVIS-LAMASTRO, V. (1990). Perceived organizational support and employee diligence, commitment, and innovation, Journal of Applied Psychology, 75, 51-59.

EISENBERGER, R., ARMELI, S., REXWINKEL, B., LYNCH, P.D., \& RHOADES, L. (2001). Reciprocation of perceived organizational support. Journal of Applied Psychology, 86(1), 42-51

EMERY, Y., \& GIAUQUE, D. (2005). Paradoxes de la gestion publique, L'Harmattan.

EMERY, Y., \& GIAUQUE, D. (2012). Motivations et valeurs des agents publics à l'épreuve des réformes, Presses de l'Université de Laval

ERIKSEN, W., BRUUSGAARD, D., \& KNARDAHL, S. (2004). Work factors as predictors of intense or disabling low back pain: A prospective study of nurses' aid. Occupational Environment Medicine, 61, 398-404.

FALKENBURG, K., \& SCHYNS, B. (2007). Work satisfaction, organizational commitment and withdrawal behaviours, Management Research News, 30(10), 708-723

GALLOIS, P. (2009). L'absentéisme : comprendre et agir, Paris : Eds de Liaison.

GELLATLY, I.R. (1995). Individual and group determinants of employee absenteeism: A test of a causal model. Journal of Organizational Behavior, 16 (5), 469-485.

GIRAUD, C. (1987). L'absentéisme : un symptôme organisationnel. Une lecture sociologique du cas d'une administration au plan régional, Sociologie du travail, 3, 323-337 
GEORGE, J.M., REED, T.F., BALLARD, K.A., COLIN, J., \& FIELDING J. (1993). Contact with AIDS patients as source of work-relates distress : effects of organizational and social support, Academy of Management Journal, 36, 157-171

GOETZEL, R.Z., LONG, S.R., OZMINKOWSKI, R.J., HAWKINS, K ., WANG, S., \& LYNCH, W. (2004). Health, absence, disability, and presenteeism cost estimates of certain physical and mental health conditions affecting U.S. employers. Journal of Occupational and Environmental Medicine, 46(4), 398-412

GOLDBERG, C.B., \& WALDMAN, D.A. (2000). Modeling employee absenteeism: Testing alternative measures and mediated effects based on job satisfaction. Journal of Organizational Behavior, 21, 665-676.

GOSSELIN, E., \& LAUZIER, M. (2011). Le présentéisme: Lorsque la présence n'est pas garante de la performance. Revue Française de gestion, 211, 15-27.

HACKETT, R.D. (1989). Work attitudes and employee absenteeism: A synthesis of the literature. Journal of Occupational Psychology, 62(3), 235-248.

HACKETT, R. D., BYCIO, P., \& HAUSDORF, P. A. (1994). Further assessments of Meyer and Allen's (1991) three-component model of organizational commitment. Journal of Applied Psychology, 79(1), 15-23.

HANISCH, K.A., \& HULIN, C.L. (1991). General attitudes and organizational withdrawal: an evaluation of causal model, Journal of Vocational Behavior, 39, 110-128.

HARRISON, D.A., \& MARTOCCHIO, J.J. (1998). Time for absenteeism: A 20-year review of origins, offshoots, and outcomes. Journal of Management, 24 (3), 305-350

HARRISON, D. A., \& PRICE, K. H. (2003). Context of consistency in absenteeism: Studying social and dispositional influences across multiple settings. Human Resource Management Review, 13, 203-225

HARRISON, D. A., NEWMAN, D. A., \& ROTH, P. L. (2006). How important are job attitudes? Meta-analytic comparisons of integrative behavioral outcomes and time sequences. Academy of Management Journal, 49, 305-325

HUVER, B. (2013). Du présentésime au travail : Mesures et facteurs explicatifs, Thèse de Doctorat de sciences économiques, Lille 1

JOHNS, G. (1994). How often were you absent? A review of the use of self-reported absence data. Journal of Applied Psychology, 79(4), 574-591.

JOHNS, G. (1997). Contempory research on absence from work: correlates, causes and consequences. International Review of Industrial and Organizational Psychology, 12, 114-173

JOHNS, G. (2003). How methodological diversity has improved our understanding of absenteeism from work. Human Resource Management Review, 13, 157-184

JOHNS, G. (2010). Presenteeism in the workplace: A review and research agenda, Journal of Organizational Behavior, 31, 519-542

JOHNS, G., \& NICHOLSON, N. (1982). The meanings of absence: New strategies for theory and research ». In Staw, B.M. \& Cummings, L.L. (Eds.). Research in Organizational Behavior, 4, 127172, Greenwich, JAI Press.

JOSEPHSON, M., LINDBERG, P., VOSS, M., ALFREDSSON, L., \& VINGARD, E. (2008). The Same Factors Influence Job Turnover and Long Spell of Sick Leave: A 3-Year Follow up of Swedish Nurses. European Journal of Public Health, 18(4), 380-385

KAISER, C.P. (1998). What do we know about employee absence behavior? An interdisciplinary interpretation. Journal of Socio-Economics, 27(1), 79-96. 
LAOUKILI, A. (2009). Les collectivités territoriales à l'épreuve du management. Connexions, 91(1), 103-121

MAHE DE BOISLANDELLE, H., \& BRUTE DE REMUR, I. (coord.), Les nouveaux défis du manager public : conduire le changement, maîtriser la gestion, dynamiser le territoire, L'Harmattan

MARTINET, A. C., \& PAYAUD, M. A. (2006). Absorption de l'incertitude, enrichissement de la stratégie et cadres intermédiaires : Une modélisation ago-antagoniste. Revue Management International, 10 (2), 29-42

MARTOCCHIO, J.J., \& JIMENO, D.I. (2003). Employee absenteeism as an affective event, Human Resource Management Review, 13, 227-241

MATHIEU, J.E., \& ZAJAC, D.M. (1990). A review and meta-analysis of the antecedents, correlates, and consequences of organizational commitment, Psychological Bulletin, 108(2), 171-194

MAYER, R. C., \& SCHOORMAN, F. D. (1992). Predicting participation and production outcomes through a two-dimensional model of organizational commitment. Academy of Management Journal, 35, 671-684

MEYER, J.P. (1997). Organizational Commitment. In COOPER, C. L. \& ROBERTSON, I.T. (Eds.), International Review of Industrial and Organizational Psychology. New York: Wiley.

MEYER, J.P., \& ALLEN, N. J. (1991). A three-component conceptualization of organizational commitment. Human Resource Management Review, 1, 61-89

MEYER, J.P., ALLEN, N.J., \& SMITH, C.A. (1993). Commitment to organizations and occupations: Extension and test of a three component conceptualization ». Journal of Applied Psychology, 78(4), 538-551

MEYER, J.P., STANLEY, D., HERSCOVITCH, L., \& TOPOLNYTSKY, L. (2002). Affective, continuance and normative commitment to the organization: A meta analysis of antecedents, correlates and consequence. Journal of Vocational Behavior, 61, 20-52.

NICHOLSON, N. (1977). Absence behaviour and attendance motivation: A conceptual synthesis, Journal of Managerial Studies, 14, 231-252

NICHOLSON, N., \& JOHNS, G. (1985). The Absence Culture and the Psychological Contract-Who's in Control of Absence?. Academy of Management Review, 10(3), 397-407

PAILLE, P. (2007). Les relations entre le soutien organisationnel perçu, les comportements de citoyenneté organisationnelle et l'intention de quitter l'organisation. Bulletin de psychologie, 490(4), 349-355

QUENNEVILLE, N., BENTEIN, K., \& SIMARD, G. (2010). Des valeurs organisationnelles à la mobilisation des ressources humaines. Revue canadienne des sciences de l'administration, 27(2), $i$ $x v i$

RANDALL, D. (1990). The consequences of organizational commitment: Methodological investigation. Journal of Organizational Behavior, 11, 361-378

RHOADES, L., EISENBERGER, R., \& ARMELI, S. (2001). Affective commitment to the organization: The contribution of perceived organizational support. Journal of Applied Psychology, $86,825-836$

RHOADES, L., \& EISENBERGER, R. (2002). Perceived organizational support : a review of the literature. Journal of Applied Psychology, 87, 698-714

ROUSSEAU, D.M., \& TIJORIWALA S.A. (1998). Assessing psychological contracts: Issues, alternatives and measures, Journal of Organizational Behaviour , 19, 679-695.

SAGIE, A. (1998). Employee absenteeism, organizational commitment, and job satisfaction: Another look. Journal of Vocational Behavior, 2, 156-171. 
SCHAUFELI, W.B., \& BAKKER, A.B. (2004). Job demands, job resources, and their relationships with burnout and engagement : A multi-sample study. Journal of Organizational Behavior, 25, 293-315

SHAPIRA-LISHCHINSKY, O., \& ROSENBLATT, Z. (2009). Perceptions of organizational ethics as predictors of work absence: a test of alternative absence measures. Journal of Business Ethics, 88, $717-734$

SMULDERS, P.G. (1980). Comments on employee absence/attendance as a dependent variable in organizational research. Journal of Applied Psychology, 65(3), 368-371.

SOMERS, M. (1995). Organizational commitment, turnover, and absenteeism: An examination of direct and indirect effects. Journal of Organizational Behavior, 16 (1), 49-58

SPECTOR, P.E., \& FOX, S. (2002). An emotion-centered model of voluntary work behavior: some parallels between counterproductive work behavior and organizational citizenship behavior. Human Resource Management Review, 12, 269-292

STEEL, R.P. (2003). Methodological and operational issues in the construction of absence variables. Human Resource Management Review, 13 (2), 243-251.

STEERS, R.M., \& RHODES, S.R. (1978). Major influences on employee attendance: A process model. Journal of Applied Psychology, 63: 391-407

THEVENET, M., \&VACHETTE, J.L. (1992). Culture et comportements, Vuibert

THEVENET, M. (2009). Manager en temps de crise, Paris, Éd. d'Organisation

THOMAS, T.T., \& GANSTER, D.C. (1995). Impact of family-supportive work variables on workfamily conflict and strain: a control perspective, Journal of Applied Psychology, 80 (1), 6-15

VAN THIELE U., LINDFORS P., \& LUNDBERG U. (2006). Evaluating different measures of sickness absence with respect to work characteristics. Scandinavian Journal of Public Health, 34(3), 247-253.

WAYNE, S.J., SHORE, L.M., BOMMER, W.H., \& TETRICK, L. E. (2002). The role of fair treatment and rewards in perceptions of organizational support and leader-member exchange, Journal of Applied Psychology, 87(3), 590-598

WEISS, D. (1979). L'absentéisme, Revue française des Affaires sociales, 10-12(4), 49-95.

WHITSTON, C., \& EDWARDS, P.K. (1990). Managing Absence in an NHS Hospital. Industrial Relations Journal, 21(4), 287-297

WOODS, S.A., POOLE, R., \& ZIBARRAS, L.D. (2012). Employee absence and organizational commitment: Moderation effects of age. Journal of Personnel Psychology, 11(4), 199-203 